Chapman University

Chapman University Digital Commons

Accounting Faculty Articles and Research

Accounting

$10-2013$

\title{
Human Economic Choice As Costly Information Processing
}

John Dickhaut

Chapman University

Vernon L. Smith

Chapman University, vsmith@chapman.edu

Baohua Xin

University of Toronto

Aldo Rustichini

University of Minnesota

Follow this and additional works at: http://digitalcommons.chapman.edu/accounting_articles

Part of the Accounting Commons, Economic Theory Commons, and the Other Economics Commons

\section{Recommended Citation}

Dickhaut, John, Vernon Smith, Baohua Xin, and Aldo Rustichini. "Human economic choice as costly information processing." Journal of Economic Behavior \& Organization 94 (2013): 206-221.

DOI: 10.1016/j.jebo.2013.08.001

This Article is brought to you for free and open access by the Accounting at Chapman University Digital Commons. It has been accepted for inclusion in Accounting Faculty Articles and Research by an authorized administrator of Chapman University Digital Commons. For more information, please contact laughtin@chapman.edu. 


\section{Human Economic Choice As Costly Information Processing}

\section{Comments}

NOTICE: this is the author's version of a work that was accepted for publication in Journal of Economic Behavior \& Organization. Changes resulting from the publishing process, such as peer review, editing, corrections, structural formatting, and other quality control mechanisms may not be reflected in this document. Changes may have been made to this work since it was submitted for publication. A definitive version was subsequently published in Journal of Economic Behavior \& Organization, volume 94, in 2013. DOI: 10.1016/j.jebo.2013.08.001

The Creative Commons license below applies only to this version of the article.

\section{Creative Commons License}

\section{(a) $\mathbb{D} \Theta \Theta$}

This work is licensed under a Creative Commons Attribution-Noncommercial-No Derivative Works 4.0 License.

\section{Copyright}

Elsevier 


\title{
Human Economic Choice as Costly Information Processing
}

\author{
John Dickhaut \\ Argyros School of Business and Economics, Chapman University \\ Vernon Smith \\ Economic Science Institute, Chapman University \\ Baohua Xin \\ Rotman School of Management, University of Toronto
}

\author{
Aldo Rustichini \\ Department of Economics, University of Minnesota
}

\begin{abstract}
We develop and test a model that provides a unified account of the neural processes underlying behavior in a classical economic choice task. The model describes in a stylized way brain processes engaged in evaluating information provided by the experimental stimuli, and produces a consistent account of several important features of the decision process in different environments: e.g., when the probability is specified or not (ambiguous choices). These features include the choices made, the time to decide, the error rate in choice, and the patterns of neural activation.

The model predicts that the further two stimuli are from each other in utility space, the shorter the reaction time will be, fewer errors in choice will be made, and less neural activation will be required to make the choice. The model also predicts that choices with ambiguity can be made more quickly and will require reduced neural activation in the horizontal intra-parietal sulcus than for choices with risk. Also, everything else being equal a larger value of certainty option in the choice will induce larger neural activation, and less experience on the part of the subject making choices will induce larger activation.
\end{abstract}

Keywords: Experimental Economics; Neuroeconomics; Economic Choices 


\section{Introduction}

We study a classical choice task collecting information on choices, reaction time and brain activation to test at a unified account of a decision maker's choice process. Our focus is on how subjects respond to different information available on the utility to them of the options offered to allocate mental effort in choice. Theories of economic choice have long ignored the fundamental role that effort can play in the decision process, though it has been hypothesized that choice requires an effort allocation.

We propose a random walk model of decision process with endogenous barriers as a function of effort that has a cost and produces benefits that vary with information quality. The model assumes that the decision maker first assesses the quality of the information available to hide, determines the evidence required to decide (in the form of a criterion or threshold to be reached), and then processes this evidence to reach a decision. The subject continuously receives a pair of signals from each choice stimulus: When enough evidence is gathered so that the number of favorable comparisons for one of the choices reaches the threshold (as in a classical random walk, or Drift Diffusion model of choice), the subject makes the choice. In summary, our model introduces choice of a costly effort into a standard Drift Diffusion model, and tests its experimental implications.

A critical element in this model is the notion of certainty equivalent. The certainty equivalent of a lottery is the monetary amount that makes the economic agent indifferent between the lottery and that amount. The certainty equivalent can be used to implement a cutoff policy: when the value of the certain amount is larger than the certainty equivalent, the subject will choose the certain amount; otherwise he will choose the lottery. The choice between two lotteries can similarly be considered as a comparison between two certainty equivalents. Thus we can think that the decision process consists of gathering information on certainty equivalents and then comparing them. It is this process that we will model and test with our experiments. 
Questions related to ours have played a prominent role in economic experiments (Allais 1953, Ellsberg 1961, MacCrimmon et al. 1980, Kahneman and Tversky 1979, and Luce 2000). Recently efforts have emerged in applying neuroscience methods to studying subjects making choices between such gambles, ${ }^{1}$ but the determination and understanding of the process involving certainty equivalent cutoffs has not been part of those inquiries. In particular we will uncover cutoffs associated with risky and ambiguous lotteries as revealed by the choices of our subjects.

Previous studies of similar tasks have however uncovered two regularities in behavior and our model provides a possible explanation. The first regularity is: the closer the options are in value, the more frequent the error in choice (i.e. the frequency of error is higher near indifference.) The first description of such a finding in choice experiments was the seminal work Siegel (1964). A related effect in general decisions is the classical "symbolic distance" effect (Moyer and Landauer 1967). Rustichini et al. (2005) recently elaborated this phenomenon for subjects in an fMRI experiment. In this case the further the choice is from indifference, the faster the reaction time. These two findings together may seem contradictory: we may observe more frequent errors close to indifference because the cost of error is less. But this explanation is difficult in light of the longer reaction time necessary to decide when the options are closer to indifference.

A second important regularity has recently surfaced in imaging studies. Rustichini et al. (2005) and Huettel et al. (2006) find that choices between ambiguous and certain amount require less processing time than choices between risky and certain amounts. Our model leads to the prediction of such differences in reaction time and also predicts that relative brain activation will be more for risky than ambiguous gambles. The intuitive reason for this is that subjects at the moment of deciding how

\footnotetext{
${ }^{1}$ For example, Breiter et al. (2001), Dickhaut et al. (2003), Huettel et al. (2005), Hus et al. (2005), and Rustichini et al. (2005).
} 
much effort to allocate in the evaluation process take into account the return to the effort, which is low in ambiguous choices, and thus terminate the process earlier.

In summary our model implies the closer to indifference an option is the more the response time, the more errors and the more brain activation. Furthermore we predict more response time and neural activation for risky than ambiguous choices. Our experimental results are consistent with this model.

\section{Background}

\subsection{Choices under uncertainty}

The economic theory of choice considers three different types of options: deterministic, risky, and ambiguous. A deterministic option is an outcome that will occur for sure, like a payment of $\$ 10$. Risky options represent known probabilities of particular deterministic outcomes, for example, a 50/50 chance at $\$ 10$ and $\$ 50$. In an ambiguous option the probabilities of the outcomes are not known by the subject. For example, the deterministic outcomes of a risky gamble can be $\$ 10$ and $\$ 50$, with no statement of the chances of those outcomes. This leaves open the possibility of a lottery ranging from a $0 \%$ chance at $\$ 10$ to a $100 \%$ chance at $\$ 10$.

In experimental economics, it is assumed that subjects come to the experiment with an ordering on dollars (more are preferred to less), and then additional attempts to understand a subject's preferences proceed from there (Smith 1982). Information about the relative preferences of a few options can be used to develop predictions about choices between other options. For example, suppose that option A is preferred to option $\mathrm{B}$; then, an option in which there is a $p$ chance $(0<p<1)$ at $\mathrm{A}$, and a $(1-p)$ chance at B will be preferred to option B, but not to option A. Also, choices between options can be used to determine distance information between preferred options. Suppose we observe that a 50/50 chance at A and B is preferred to a 50/50 chance at C 
and D. Then if we also observe that a 50/50 chance at $A$ and $C$ is preferred to a 50/50 chance at $\mathrm{B}$ and $\mathrm{D}$, we can conclude that $A$ is preferred to $D$.

Such determinations of the implicit numerical properties of these stimuli were part of the constructions of Davidson et al. (1955) and were used to derive utility representations of various objects such as books, records, and grades of students as well as cigarettes for prisoners (Siegel et al. 1964). One point of the current paper is to test the hypothesis that evaluation projects options to an ordered line segment that can be interpreted as representing utility in economic choice studies (similar to numerical comparisons studied in Dehaene 1999). Smith and Walker (2007) model choice as a productive activity, with returns and costs of making the choice taking the form of an additively separable utility function. A similar approach is outlined in several different approaches to behavioral economics (see Camerer and Hogarth 1999, Johnson and Payne 1985).

\subsection{Findings from early studies of choice}

As we mentioned earlier, experimental analysis of choice has identified and replicated two important regularities of the choice process.

\section{Inconsistency near cutoff}

Early studies of choice involved comparisons between deterministic outcomes. Thurstone (1931) outlined a methodology in which a person was asked to choose between two-tuples at a time, for example, ( 2 hats, 4 pairs of shoes) versus ( 4 hats, $x$ pairs of shoes), where $x$ was varied. Using this approach creates an estimated cutoff point $z$ such that for an $x$ below $z$, (2 hats, 4 pairs of shoes) will likely be preferred to (4 hats, $x$ pairs of shoes), and for $x$ above $z$, (4 hats, $x$ pairs of shoes) will likely be preferred to (2 hats, 4 pairs of shoes). Thurstone's data reveal that subjects tend to 
reveal more noise in choices relative to a cutoff when $x$ is close to the cutoff point (see also Mosteller and Nogee 1951 and Edwards 1954).

\section{Increased reaction time near cutoffs}

This is a very early finding. For example, Mosteller and Nogee (1951) measured reaction time in choosing between risky gambles during choices and were able to determine that the closer to indifference a subject was, the higher the reaction time tended to be. The net result in these early studies is that when distance (here, distance in utility space) comes into play consistency and reaction time are affected. Recently, efforts have emerged to apply neuroscientific methods to studying subjects making choices between gambles, but the determination and understanding of the process regarding cutoffs in choices has not been elaborated neuronally.

We will uncover cutoffs associated with risky and ambiguous lotteries, as revealed by the choices of our subjects.

\subsection{Neural studies}

A variety of results reviewed below (for human and nonhuman primates, in particular for the processing of numerosity) suggest a general model of the decision process which can be considered an extension of Ratcliff's random walk model of memory retrieval (Ratcliff 1978). We propose that such a process governs choices among economic options, as those made by subjects in our experiment. Our subjects had to choose between a lottery and a certain amount. A lottery specifies a payment made to the subject depending on the outcome of a random device, which could be

risky or ambiguous. When a subject considers, say, a risky lottery, he can tentatively assign to it a corresponding certain utility amount that is located on the ordered real line. When he has to choose between this lottery and a certain amount, the subject can simply compare the utility of the risky lottery and the utility of the certain amount. 
The choice between the two lotteries, certain and uncertain, is now determined by the comparison of these two values.

Neural studies have focused on the impact of administered reward and isolated areas of the nucleus accumbens and mesial prefrontal cortex associated with the processing of gains and the probability of gains, respectively (Knutson et al. 2001). Kable and Glimcher (2007) found evidence of sensitivity to estimated subjective value (as opposed to objective rewards) in the striatum, mesial prefrontal cortex, and posterior cingulate cortex in a discounting task.

Using positron-emission tomography (PET), several studies of choice suggested that the type of gamble (ambiguity, risk, and certainty) leads to differential activation in the parietal, frontal, and orbital frontal areas (Rustichini et al. 2005). For responses to gains and losses, Tom et al. (2007) showed extensive dopaminergic engagement as well as prefrontal involvement for individual subject analysis.

Two studies focused on the ambiguity, risk, and certainty distinctions we adopt in this paper. Hsu et al. (2005) inferred differences in activation between risk and ambiguity in the amygdala and lateral orbital frontal cortex, whereas Huettel et al. (2006), using stimuli similar in construction to those used in the current study, found that risky stimuli activate the parietal area, while ambiguous stimuli differentially activate the lateral prefrontal cortex.

Our model of human economic choice is informed by evidence on the processing of numerical magnitudes (see Dehaene 1999). Consider, for instance, a very simple number comparison task: a human subject is shown a number on a screen and is asked to press a left button to indicate that the number is less than 55 and a right button to indicate that the number is greater than 55. An interpretation of the process is as follows. The human brain forms a spatial representation of numerical magnitudes. A comparison stimulus is presented on a screen activates areas distinctly responsible for making comparisons of the spatial representations: the bilateral 
horizontal segment of the intraparietal sulcus, the left angular gyrus, and the bilateral posterior superior parietal lobe (Dehaene et al. 1990). The projections in terms of neuronal activation of the comparison stimulus are registered on "greater than 55" counters and "less than 55 counters." Which counter hits a barrier first will determine whether the subject indicates that the number is greater than or less than 55 .

Several of the main modeling results in economic choice in this study are paralleled by results with numerical magnitudes. In studies of numerosity, there are distance and inconsistency effects, just like in the early studies on choice. The distance effect with numbers has been replicated many times with humans and nonhuman primates. The underlying explanation of such phenomena is that number symbols, such as Arabic or Roman numerals, or collections of dots (in the case of monkeys) are transformed to associative cortex, and semantic representation is made downstream (Tudusciuc and Nieder 2007). Such representations are noisy. For example, chimpanzees trained to match to sample will most frequently match 3 dots with 3 dots previously shown; 2 and 4 dots are less frequently matched to 3; and 5 dots are rarely matched, if ever. The explanation for the human judgments is that humans also work with a noisy representation, even of certain amounts, and that they map directly from the noisy representation to motor actions so that such actions are noisy in response to experimental stimuli (Tudusciuc and Nieder 2010).

A verification task such as answering true or false to the statement $3+4=12$ is generally construed to require more than one operation (Logan and Zbrodoff 2003). In a verification task, addition and comparison are two such operations. With no constraints, reaction time is larger in such tasks and often different from the sum in both tasks, indicating that the subject adjusts reaction time as a consequence of the context of the numerical problem. Thus task differences in using numbers can produce different reaction times, much like tasks using ambiguous and risky stimuli produce different reaction times. 
Generally, several forms of study, lesion research, single-cell recording, PET, and functional magnetic resonance imaging (fMRI) tend to implicate the inferior parietal cortex and the frontal lobe in numerical operations (Dehaene et al. 2003). The most frequently implicated locations for neuronal involvement in numerical operations are the left and right inferior parietal sulci. In studies of patients with lesions in the general parietal area, approximation in division and subtraction tasks is severely hampered. Single-cell recordings implicate specific neurons associated with numerosity. For example, detection neurons sensitized to the number 6 have been reported in association cortex in an anaesthetized cat. Monkeys' superior parietal lobules are tuned to counting the number of muscle movements of monkeys as well as a distance effect (Sawamura et al. 2002). In a match-to-sample test using monkeys, inferior parietal and frontal lobe activation showed a larger proportion of neurons sensitive to numbers, and sensitivity in the inferior parietal area preceded sensitivity in the frontal area (Nieder and Miller 2003). These findings generally provide support for connectivity in nonhuman settings with respect to number assessment. The earliest imaging study of numerical processing supports an important role for the intraparietal sulcus as well as the prefrontal cortex in a subtraction task (xenon was injected into a carotid artery to detect behavior of single photons; Roland and Friberg 1985). The original result was replicated using PET and, later, was used to specifically isolate responses in a subtraction task to isolate parietal and prefrontal activation.

\section{A Theoretic Framework and Hypotheses}

The model is an extension of Dichhaut et al. (2009), which combines the main ideas from the theory of signal detection and of the random walk models of decision, and then extends them to the realm of economic decisions. We explicitly introduce two additional elements. First, since we are interested in what level of brain activity is associated with risky and ambiguous tasks, we introduce a variable, the quality of the 
information environment in which subjects are making decisions. Second, we introduce endogenous barriers as a function of effort that varies with information quality into the random walk model of decision process. We assume that prior to any choice subjects' behavior can be specified in terms of an economic model that incorporates a selection of effort to generate internal (neuronal) information about which choice to make (the left or right alternative on a computer screen). By economic, we mean a model that formally represents prior information, conditional information on outcomes, preferences on outcomes, and separable preferences on effort. Furthermore, information has a definite impact on beliefs through a mechanism that organizes processing. In an economic model, this is Bayes' theorem.

\subsection{Theoretic Framework}

The subject has to choose one action out of a set $A$. If as in our experiment he is choosing between two lotteries one on the left and one on the right, we can describe the action set as $A=\{L, R\}$. He is trying to evaluate which of the two lotteries has a larger utility. His payoff $V$ will depend on the action and a state of nature that is unknown, $\theta \in \Theta$. He does not know the utility of the two options, so the unknown state is the value of the two utilities $\theta=\left(\theta_{1}, \theta_{2}\right)$. If the value $\theta$ was known, then the optimal choice would be clear: choose the lottery with the largest value. He does not know the value, however, so his choice has to rely on a noisy signal, $y \in Y$, on the comparative value of the two options. We consider this comparative value to be represented by a function with value $A\left(\theta_{1}, \theta_{2}\right)$ at $\left(\theta_{1}, \theta_{2}\right)$ This function is assumed to be strictly increasing in the first component and strictly decreasing in the second, and is such that for every $\theta_{1}$ and $\theta_{2}: A\left(\theta_{2}, \theta_{1}\right)=-A\left(\theta_{1}, \theta_{2}\right)$. For simplicity, we consider the case where the function depends on the difference: $A\left(\theta_{1}-\theta_{2}\right)$ as an illustration in the following analysis. The subject has an initial belief on the states of nature, $\mu$, the prior probability of a particular utility pair from the set of possible lotteries, which is 
symmetric: $\mu\left(d \theta_{1}, d \theta_{2}\right)=\mu\left(d \theta_{2}, d \theta_{1}\right)$.

The signal set is $\mathrm{Y}=\{-1,1\}$ : a 1 (respectively a -1 ) is a "match" (respectively "mismatch"). We assume that this probability is only dependent on the comparative value $A(\theta)$, and that the signal provided by $P$ is a location signal. The signal $y$ can be considered as resulting from the brain processing input generated by the experimental stimulus. Figure 1A demonstrates such a process. This graph shows the conditional distribution of the comparison metric $A$ when the real difference $\theta_{1}-\theta_{2}$ is located on the positive horizontal axis. Note that $A$ yields a +1 if $A$ is bigger than 0 and a -1 otherwise. Given that $A$ is an estimate of the difference $\theta_{1}-\theta_{2}$, then a $y$ of 1 is said to be a match. Two features of $A$ are that it is noisy and that it is only indirectly available for downstream purposes by the brain through the creation of the signal $y$. The signal $y$ depends on the level of effort $e \in E$ that the subject chooses: given the true state $\theta$ and the effort $e$, the probability of $y$ is denoted by $P_{\theta}(y ; e)$. Specifically the probability of a match is given by:

$$
P_{\theta}(1: e)=\int_{-\pi(n) e A(\theta)}^{+\infty} f(x) d x
$$

for a given density function $f$. The effectiveness of the effort is increased by a function $\pi$ of $n$, a measure of subjects' experience in the task. We assume that the function $f$ satisfies: 1) $f(x)=f(-x)$, i.e., no differential treatment of options on $L$ and $R ; 2)$ if $x>y>0$ then $f(y)>f(x)$, i.e., the probability decreases as we move away from 0 . In light of having exerted the processing effort $e$, the decision maker considers choosing each action, $L$ and $R$, and picks the action that has the highest net expected utility. The optimization problem for given effort, and observed signal is:

$$
\max _{a \in A} \int_{\Theta} \mu(\theta) P_{\theta}(y ; e) V(\theta, a) .
$$

The subject decides on the optimal level of effort. The provision of effort in observing and processing the signal is costly. The cost is influenced by the quality of information that he has available. In our experiment the subjects had to choose when 
the quality of information was high (when the lottery is risky, and the probability of the two outcomes is known) or low (when the lottery is ambiguous). We summarize the quality of information with a variable $q$ which can be 0 (ambiguous lottery) or 1 (risky lottery); so a higher value of $q$ corresponds to better information. Putting these elements together, the cost is $C(e ; q)=\gamma(q) c(e)$. We assume that the function is decreasing in the quality of information $q$, and that the function $c$ is convex. The optimization problem has the form:

$$
\max _{e \in E}\left(\int_{Y} \max _{a \in A} \int_{\Theta} \mu(\theta) P_{\theta}(y ; e) V(\theta, a)-C(e ; q)\right) .
$$

One can prove, given the symmetry assumptions on the beliefs and the signal, that the optimal action given the signal $y=1$ is $l$. Let $\hat{a}(y)$ be the optimal action with signal $y$, $W(e \mid y)=\int_{\Theta} \mu(\theta ; y, e) V(\theta, \hat{a}(y))$ and $W(e)=\int_{Y} P_{\mu}(y) W(e \mid y)$. The functions $W(\cdot \mid y)$ and $W$ are increasing in $e$, and the derivative is decreasing, for fixed $n$. Also, for every $e$, $W(e)=\frac{1}{2} W(e \mid 1)+\frac{1}{2} W(e \mid-1)$ and $W(e \mid 1)=W(e \mid-1)=W(e)$.

Optimal effort is determined equating marginal returns and marginal cost of effort.

This process proceeds as a random walk model. The essential elements of the model are a space of evidence, a stochastic process on this space (reflecting accumulated evidence), and a barrier. The process stops when the barrier (high or low) is reached, and a decision corresponding to the barrier is made. The decision process has been reduced to a random walk on a space and a level of evidence described by a barrier.

The barrier in the random walk model of choice can be interpreted as expressing the level of satisfactory evidence which is considered necessary to take a decision. If this level is independent of the quality of the information, then prediction on response time is clear: The conditions in which the information is inferior would require a longer time to reach the same level of satisfactory evidence; however the informationprocessing device (the brain) should consider also that inferior information gives a 
smaller incentive to wait while observing. For example, if the signal observed provides no information at all, then clearly the optimal policy is to decide immediately, since observing the signal is not going to improve the quality of the decision. In this case the barrier is reached very close to the starting point of the process. Thus a less informative signal has the effect of reducing the level of evidence considered necessary before the information processing stops and decision is reached.

In summary, given a barrier, our model of the decision process has two additional components: first, the process reduces the complex information describing two economic options to a numerical value, the utility of each option. The second performs the comparison between these two quantities and determines, possibly with an error, the larger of the two. The comparison in this second step is well described by a random walk of decision with endogenous barriers as a function of the chosen effort. This theoretical construction allows us to discipline our examination of the data.

\subsection{Hypotheses}

The comparative value $A$ reflects some level of effort. Note that if there were no effort, the distribution would not be distinguishable from an improper uniform distribution on $\{-\infty, \infty\}$, meaning that a $y$ of 1 would not distinguish the left- and righthand options to the subject. The effect of increasing effort is captured by Figure 1B. The impact of effort is to make $y$ more discriminating in the sense that when the effort is high, a $y$ (integrate $A$ over the positive domain) of 1 is more likely to occur for a positive $\left(\theta_{1}-\theta_{2}\right)$. Asymmetric construction holds for a negative $\left(\theta_{1}-\theta_{2}\right)$. It will be the case that as effort increases, the quality of the signal $y$ increases in the sense that $y$ will have a higher probability of being 1 given that $\left(\theta_{1}-\theta_{2}\right)$ is any positive magnitude. This implies that other things being equal, an increase in effort will decrease the time to respond and reduce the error, which is defined as the event in which the subject chooses the option with smaller utility. 
We assume that the signal derived from the choices will improve, and the expected utility will improve at a lesser rate for the same level of effort as the experience of the subject increases. This is shown in the marginal benefit curve in Figure 1C. An increase in experience (the variable $n$ ) has an increasing returns and a substitution effect: the first increases optimal effort, the second decreases it. For experienced subjects, if the second effect is dominant, the resulting effect is a reduction of the effort. On the other hand, the subject can, in a particular choice, face higher- or lower-quality information. The lower-quality information will demand less effort to process (there is less of it) and result in a faster rate of extraction, and hence the optimal level of effort will decrease. Thus an increase in the quality of information (the variable $q$ ) has the effect of an increase in the effort, because it shifts the marginal cost function down. This leads to a faster evidence accumulation. However a higher quality of information also means that the subject will set a higher threshold for the level of evidence accumulated to reach a decision. When this latter effect dominates, it takes longer to respond.

Expression (3) represents updated beliefs by the subject after knowing $y$ and having expended effort $e$. The magnitude of the certainty option in the decision, $c$, is related to the initial attention the subject pays to the choice (as opposed to effort to process the choice). There is substantial literature on magnitude of the payoffs and the relationship of the payoffs to performance. Research began with the work of Davidson et al. (1955), carries through to the discussion of salience by Smith (1982), and finds numerous other examinations by such authors as Harrison (1989, 1994), Binswanger (1980), and Khaneman and Tversky (1979), although results in this literature are not always in agreement. In the current model, we capture the proposition that a higher magnitude attracts more attention so that a subject, when he or she sees a $\$ 15$ option, will attend less than when he or she sees a $\$ 30$ option. Traditionally, attention has been 
a psychological variable, but we argue that attention has a definite effect on the choice model through its effect on priors. Specifically, the assumption we make is that a higher certainty option means that the subject entertains a higher probability for all the alternative pairs under consideration. The magnitude of $c$ will, via attention, affect the effort level chosen. Therefore we expect that the increase (decrease) in the value of the certain amount (the variable $c$ ) shifts upwards (downwards) the marginal benefit of the effort, leading to an increase (decrease) in effort.

Define the distance between $\theta$ and $\tilde{\theta}$ as the absolute value of the difference between the utility of the two options (e.g., the variable $|A(\theta)-A(\tilde{\theta})|$ ). As this distance increases, the probability of receiving a "match" signal increases. This speeds up "right" evidence accumulation, and has the effect of reducing error and time to respond.

We summarize the main predictions of our model:

1. An increase in experience shifts the derivative of the benefit function down and leads to a reduction of effort.

2. An increase in the quality of information similarly shifts the derivative of the cost function down, leading to an increase in effort.

3. An increase in the value of the certain amount has the effect of an increase in effort.

4. An increase in the distance in utility between the two options decreases the time to respond.

5. An increase in the distance in utility between the two options decreases the error rate.

\section{Experimental Design and Implementation}




\subsection{The choice task}

Each experimental session consisted of three successive blocks. Each block consisted, in turn, of 14 periods. In each period, the subject had a choice between two options. One of the options was a lottery, while the other was a certain amount. A lottery consisted of two monetary prizes and a probability for each. Seven of the lotteries were risky, and seven were ambiguous. In a risky lottery, a pie drawing represented the probability of the two outcomes; in the ambiguous choice, no information was given about the probability. Display on the left and on the right side of the screen of the certain and uncertain outcome were balanced.

Figure 2 illustrates the visual presentation of choices. The display of the certain amount and the uncertain lotteries are clearly differentiated. A lottery is described by pie chart illustrating the probability of the two events. The ambiguous lotteries are presented with a pie chart reporting the colors that correspond to win and loss, but no split between the two colors, to emphasize that the probability is not specified and may correspond to any feasible partition of the circle. This device emphasizes the lack of information on the probability. The monetary prizes for the uncertain lotteries were, in every case, 50 and 10 dollars; the values of the certain lotteries were 15, 17, 20, $25,25,27$, and 30 dollars.

Similar designs to study ambiguity have also been used in Huettel et al. (2006) and Levy et al. (2010). Values of the lottery outcomes and certain amounts were chosen in line with previous studies (see Dickhaut et al, 2003, Rustichini et. al, 2005). Appropriate modifications were made to address the specific hypotheses tested here: The certainty amounts were chosen in such a way that the indifference point between a risky (ambiguous) lottery and a certain amount falls between the lowest and highest certainty amounts. These choices enabled us to investigate such issues as error rate and reaction time. 


\subsection{Feedback}

In the first and third blocks, the subject had no feedback after his or her choice on the outcome of the random draw; in the second block, the subject was informed of the outcome after making a choice. In the first and third blocks, the period began with the display of the choice for $5 \mathrm{~s}$. During this time, the subject could only observe the available options. At the end of the 5-s interval, a cue on the screen signaled to the subject that he or she could choose one of the two options (GO signal). The selection of the choice was made by pressing one of two buttons on a box; the subject had no time restriction. After the subject's choice, a period of rest followed, of jittered length (mean $=13,12 \mathrm{~s}, \mathrm{SE}=0.037 \mathrm{~s}$, in which the subject had no task to perform (see Figure 2 for the time line of blocks 1 and 3).

The design of the second block was identical up to the point at which the subject made his or her choice. After that, the pie chart displayed the probability of the two choices in the ambiguous case. Then, in both the risky and the ambiguous case, a spinner rotated and then stopped in one of the two pie sectors, indicating the outcome for the subject. The spinner had the shape of an arrow, rotating around the center of the pie, displaying the probability of the two outcomes. This entire step lasted $23 \mathrm{~s}$. After the spinner step, the subject waited for a period of $13 \mathrm{~s}$, after which the next period began. The sequence of events that we have described after the choice was the same, independently of the choice made by the subject, so the subject would know the outcome of the uncertain choice even if he or she had chosen the certain amount. Captions of Figure 2 report the detail timing of the presentation of choices and feedback.

\subsection{Subjects}

Fourteen subjects were scanned while performing the task. The subject communicated his or her choices through a MR-compatible button box; choices were 
presented on a screen located behind the subject, who could see them through a mirror attached to the head coil. The study had the approval of the Institutional Review Board at the University of Minnesota. Subjects were students recruited at the university (average age 22.5 years, standard deviation 2.95). Seven subjects were female. The average payment to subjects, including the participation payment and the money gained in the task, was $\$ 83$.

Subjects in the experiment had to choose in every period between a lottery and a certain amount. The lottery could be risky or ambiguous, depending on whether the probability over outcomes was clearly specified. We recorded participants' response times measured in seconds from the moment the cue signaling that choice could be made appeared on the screen until a choice was made. For each subject, we computed a cutoff value as the empirical estimate of the indifference point. This was defined to be a value that would give the observed number of choices of the certain amount made by the subject. The empirical cutoff may be different in different blocks, and for ambiguous and risky choices, so it was computed separately, conditional on the block and the quality of information. On the basis of this empirical cutoff, we assessed the variable error. More precisely, the error was defined to be 0 for a given trial if the value of the certain amount was less than the empirical cutoff (so that the subject should choose the lottery) and the subject chose the lottery; it was defined to be 1 if the subject chose the certain amount. The error was defined similarly for the case in which the certain amount was larger than the cutoff.

\section{Results}

\subsection{Choices}

Subjects were less willing to choose the ambiguous lottery than the risky one. The logit regression of the choice of the certain amount supports this conclusion: the coefficient of the variable ambiguous (equal to 1 if the lottery was ambiguous) is 
positive and significant ( $p$-value 0.052 ). The odd ratio of choosing the certain amount is 1.56. Similarly, the value of the coefficient of the certain amount itself is positive and significant ( $p$-value $<0.001$ ). The odd ratio is 1.47 . Finally, the number of cumulative choices made has a borderline significant $(p$-value $<0.067)$ and small sized effect of reducing the probability of choosing the certain amount (odd ratio 0.98).

\subsection{Error rate}

A first prediction of our model is that the probability of an error will increase as the difference in value between the two options decreases; that is, the difference between the estimated cutoff and the certain amount decreases. Figure 3 plots the distance from the cutoff against the error. The number of errors approaches zero as the distance from the cutoff reaches 14 . Details of the calculation of the confidence bands and path estimation are provided in the Dickhaut et al. (2009). Table 2 shows that there is also an effect of the cumulative number of choices made ( $p$-value $<0.067)$. The odd ratio is 0.95 .

\subsection{Response time}

Our model leads us to predict two additional important regularities concerning time to response. The first is that the response time decreases as the distance between the value of the certain amount and the cutoff increases. We measure the difference between the certain amount and the cutoff value of that subject and graph such differences against the response time for that choice. There is a strong tendency for the response time to decline with the distance from the cutoff point of the certainty amount, for values both larger and smaller than the cutoff.

The second prediction is that risky choices require longer response times than ambiguous choices. We tested this hypothesis with a panel data analysis of the response time for each choice against three independent variables: the experience of 
the subject (measured by the total number of choices made up to that point, cumulated choices), ambiguity (equal to 1 if the choice was an ambiguous choice, and 0 if the choice was risky) and the value of the certain amount. The estimated coefficients are all significantly different from zero. An ambiguous choice reduced the time to reach a decision $(p=0.051)$. Also the number of cumulative choices made reduced the time ( $p$ $<0.001)$.

\subsection{Brain imaging}

We examine the overall decision process by contrasting the neural activity leading to the decision with neural activity after the choice has been made, in all trials, in all blocks. Figure 5 (top) shows the areas that were significantly more active during the display period leading to the decision than during the after-choice period (orangeyellow clusters). Two main areas of interest appear: the intra-parietal sulci (IPS), both in the left and right hemispheres, and the Medial Frontal Gyrus (MFG), extending to the supplementary motor area and the anterior cingulate cortex.

Figure 5 also shows areas more active in the after-choice period than during the display period (blue clusters). The strong activation in this contrast was observed in the orbitofrontal cortex (OFC), which is part of the system responsible for evaluation. This finding suggests that in this task evaluative processes occur after the choice has been committed. Padoa-Schioppa and Assad (2006) have shown activation in afterchoice in some neurons in area 13 of the OFC of the monkey that is larger than the activation occurring at the choice stage. The event-related average of the activation around the onset of the event confirms the results of the analysis we have presented. The MFG and IPS clusters identified previously co-vary in a similar manner, increasing at display and decline in after-choice, while the OFC cortex varies in the opposite way (see Figure 5, bottom). 


\subsection{Tests of the activations predicted by the theory}

Our model predicts a differential effect of the nature of the choice (ambiguous versus risky) on brain activations: the relative lack of information in the ambiguous choices will result in a lower barrier to cross at the moment of the comparison so that a given certainty option will require less activation before the barrier is crossed in the ambiguous case than the risky case. To test this hypothesis we determined the relatively most active regions at display when the subject is facing ambiguous choices and compared them with the relatively most active regions when he is facing risky choices. The results are reported in the top panel of Figure 5. The pattern of activation is similar in the two conditions, but for both clusters of activation (IPS and MFG), the risky choices generate more activation than the ambiguous choices.

In the bar graphs in Figure 6 (bottom), we assess the impact of experience, distance of the choices, quality of information, and the level of the certainty amount on the percentage change of the BOLD signal during a trial. The graphs display the average value of the coefficient of the four variables (distance of the certain amount from the cutoff, certain amount, number of choices made by the subject, and ambiguous choice), with one standard error superimposed. We assess coefficients for the four clusters identified in Figure 6 (top): right and left IPS, MFG, and OFC. The coefficients were obtained by first estimating the percentage BOLD change (PBC) for each of the 42 trials (the experiment had three blocks of 14 trials each), obtained by taking the event display in each trial as a distinct predictor. We then ran a regression on PBC with respect to the following variables: distance of the certain amount from the cutoff, certain amount, ambiguity, and number of choices made by the subject. The four variables are normalized to have range between 0 and 1 so that the coefficients are directly comparable. The signs of the coefficients are as predicted by the model. Ambiguity has a negative effect. In addition, experience reduces the activation, as indicated by the negative value of the coefficient for the number of choices made. 
The effect of the distance from the cutoff is negative: when the value is higher than the cutoff, the activation is reduced. This occurs in all four regions, including the OFC, in a significant way. The effect of the certain amount is positive. Both coefficients are strongly significant for the two IPS regions ( $p=0.0001$ for the left IPS).

\section{Conclusions}

Theories of economic choice have long ignored the fundamental role that effort can play in the resolution of the choice process. Smith and Walker (2007) explicitly model effort in the choice process. Their perspective is also reflected in the work of Camerer and Hogarth (1999), and Johnson and Payne (1985). The current study hypothesizes that effort enter into choice, has a cost and a benefit that vary with experience, information quality, and similarity of the stimuli. These effects are confirmed in measurements of blood flow, reaction time, and noise in choices in a way that is consistent with economic theory.

The theory uses as underpinnings work on numerical stimuli and earlier neuroeconomics and more generally neuroscience work (Dehaene et al. 1998). We have assessed if the numerical properties associated with the intra-parietal sulcus provide a locus for representing objects of choice as presented in the classical choice task. We find evidence for reduced reaction time and inconsistency in choice and activation the further the inferred distance on the ordered line segment choices are from each other. Furthermore, we postulate that it is possible, and demonstrate the possibility, to reduce reaction time and activation for ambiguous choices.

A number of neuroscientific studies are consistent with the calculational properties of the horizontal intraparietal sulcus playing an integral role in choice. Shadlen and Newsome (1996) found that areas in LIP encode differential counts of representations of movement in MT. Yang and Shadlen (2007) showed monkeys different shapes that implied a likelihood that a gaze right would result in a reward. 
They found evidence of the ability to add and subtract log likelihood information in the intraparietal sulcus.

We postulate that the numerical representations of choices are coded noisily in the intraparietal sulcus; we do not find that all processing occurs there. A parallel study with single-cell recording in monkeys suggests that numerical tasks can be distributed to other brain areas for analysis. For example, it has been found that when monkeys are matched to sample in a number-of-dots task, activation first occurs in the parietal lobe, but at a later time, the dorsolateral prefrontal cells are activated. Thus it is reasonable that this type of behavior is going on in our task during the display stage and that signals are being distributed from parietal to dorsolateral prefrontal. This difference could be producing the differential activation for ambiguous stimuli isolated by Huettel et al. (2006), although their level of time stamping is not achievable in our task. Another aspect of the Huettel et al. task that differs from the current task is that an explicit question mark symbol is placed on the ambiguous stimulus; in the current task, there is no such process of indicating ambiguity (see Figure 2). A more thorough understanding of time sequencing of tasks may lead to an equivalent representation of the utility of choices in distinct areas such as those uncovered by Kable and Glimcher (2007). 


\section{Appendix: Imaging methods and parameters}

\subsection{Data acquisition}

During the entire experiment, the subject was lying in supine position in the bore of the scanner, and communicated his choices through a button box. Choices were presented on a screen located behind the subject, who could see them through a mirror in the head coil. A 3-Tesla whole body MR system (Magnetom Trio; Siemens Medical Center, Erlangen, Germany) at the Center for Magnetic Resonance Research at the University of Minnesota was used for image acquisition.

Before the functional run, 144 or 160 (depending on the subject's head size) FLASH images were acquired in slices of 1-mm thickness in sagittal plane, with a field of view (FOV) of $256 \times 256 \mathrm{~mm}$ and a matrix size of $256 \times 256$ lines, giving a spatial resolution of $1 \mathrm{~mm}^{3}$ for the anatomical volume. The TR was $20 \mathrm{~ms}$, the TE was $4.7 \mathrm{~ms}$, and the flip angle was $22^{\circ}$.

After that, a whole brain functional MRI (fMRI) was performed using an echoplanar imaging sequence (EPI) measuring the blood oxygenation level-dependent (BOLD) signal. A total of 30 -38 functional slices per volume were acquired for each subject. The slices had 3-mm thickness, acquired in transversal plane, in a field of view of 192 x $192 \mathrm{~mm}$. No gap separated the slices. The TR was 2,000 ms, the TE was $23 \mathrm{ms,}$ and the flip angle was $90^{\circ}$. The matrix size was $64 \times 64$, the resolution $3 \times 3 \times 3 \mathrm{~mm}$.

\subsection{Preprocessing of fMRI data}

BrainVoyager QX version 2.1 (Brain Innovation, Maastricht, The Netherlands) software was used for fMRI data preprocessing and analysis. The two-dimensional images of every subject were preprocessed to correct for motion artifacts, with a threshold of 3-mm movements in any direction. Also, a correction for differences in slice scan time acquisition and for temporal linear trends was used. The functional 
images were then used to construct a three-dimensional (3D) functional volume for every subject and every run. Spatial smoothing was performed by using a Gaussian full width at half maximum (FWHM) kernel of $7 \mathrm{~mm}$. The 3D functional volume was then aligned with the corresponding 3D anatomic volume. Both were then normalized to standard Talairach space.

The statistical parameters of the model were computed voxel-wise for the entire brain, and activation maps were computed for various contrasts between the predictors. Data were convolved with a 2-gammas hemodynamic response function with the following parameters: onset displacement, 1 TR; response to undershoot ratio, $6 \mathrm{~s}$; time to peak of the positive function, $5 \mathrm{~s}$; of the negative function, $15 \mathrm{~s}$.

\subsection{Statistical analysis of imaging data}

We used a rapid event-related design to analyze the data in our experiment. We used both categorical and parametric models for the analysis. WE discuss each clas of models separately.

Categorical models: In each block, we defined temporal events based both on the task and subjects' reaction to the stimuli. In each block, we had predictors describing (i) presentation of choice, with onset time at the moment in which the choice was displayed on the screen (ii) the decision interval, and (iii) an after-choice period modeled as a $2 \mathrm{~s}$ box-car function after the decision was communicated by the subject. Finally a (iv) feedback interval was used when the outcome of the random event was provided to the subject. These predictors were further categorized depending on the block in which they occurred, the type of trial (risky vs. ambiguous), and the subject's choice (certain vs. uncertain).

The predictors listed in the previous paragraph were entered as fixed factors in a single-subject general linear model (GLM), and then the parameters of this GLM model were subsequently entered into a second level of analysis corresponding to a 
random-effect GLM model, which was used for group analysis (Penny and Holmes 2003). The statistical parameters of this latter model were estimated voxelwise for the entire brain, and activation maps were computed for various contrasts between the predictors. The criteria used to display the activation maps for the main GLM model were as follows: a cluster size of 100 adjacent significant voxels and a statistical threshold for each voxel in the cluster of $p<0.00005$, corresponding to a minimum $t$ value of 5.93 .

Parametric models: A second series of GLM models was constructed using the categorical predictors from the previous GLM models and by adding parametrical covariates based on subjects' performance and characteristics of the choice. Four variables were used: (i) distance of the certain amount from the cutoff estimated for the subject in behavioral data (ii) certain amount (iii) number of choices made by the subject until that moment, to take into account possible learning, and (iv) an indicator for ambiguous choice.

\subsection{Presentation of the analysis of imaging data}

The criteria used to display the activation maps for these secondary GLM models were as follows: a cluster size of a maximum 100 adjacent significant voxels and a statistical threshold for each voxel in the cluster of $p<0.005$, corresponding to a minimum $t$-value of 3.38 .

In the second stage of the analysis, the clusters obtained as a result of various contrasts in the first stage were defined as regions of interest (ROIs), and further GLM analyses were performed separately for each ROI to compute the percentage of signal change or to correlate imaging data with the behavioral measures. These latter analyses were performed taking into account the time course of the signal averaged over all voxels in the ROI for each subject, and they were performed using SPSS (SPSS Inc., Chicago, IL) and Stata (StataCorp LP, College Station, TX). 


\section{References}

Allais, M., 1953. Le Comportement de l'Homme Rationnel devant le Risque: Critique des Postulats et Axiomes de l’Ecole americaine. Econometrica 21: 503-546.

Binswanger, H.P., 1980. Attitudes toward risk: Experimental measurement in rural India. Am J Agric Econ 62: 395-407.

Breiter, H.C., Aharon, I., Kahneman, D., Dale, A., Shizgal, P. 2001. Functional imaging of neural responses to expectancy and experience of monetary gains and losses. Neuron 30:619-639.

Camerer, C.F., Hogarth, R.M., 1999. The effects of financial incentives in experiments: A review and capital-labor-production framework. J Risk Uncertainty 19: 7-42.

Davidson, D., Siegel, S., Suppes, P., 1955. Some experiments and related theory on the measurement of utility and subjective probability. Stanford: Applied Mathematics and Statistics Laboratory, Stanford University.

Dehaene, S., 1999. The number sense: How the mind creates mathematics. Oxford UP.

Dehaene, S., Dupoux, E., Mehler, J. 1990. Is numerical comparison digital? Analogical and symbolic effects in two-digit number comparison. J Exp Psychol 16: 626-641.

Dehaene, S., Lambertz, G., Cohen, L., 1998. Abstract representations of numbers in the animal and human brain. TINS 21:355-361.

Dehaene, S., Piazza, M., Pinel, P., Cohen, L., 2003. Three parietal circuits for number processing. Cognitive Neuropsychology 20: 487-506.

Dickhaut, J., McCabe, K., Nagode, J.C., Rustichini, A., Smith, K., Pardo, J., 2003. The impact of the certainty context on the process of choice. Proc Natl Acad Sci USA 6: 3536-3541.

Dickhaut, J., Rustichini, A., Smith, V., 2009. A Neuroeconomic theory of the decision process, Proc Natl Acad Sci USA 106 : 22145-22150.

Edwards, W., 1954. The theory of decision making. Psychol Bull 51: 380-417.

Ellsberg, D., 1961. Risk, ambiguity, and the savage axioms. Q J Econ 75: 643-669. 
Harrison, G.W., 1989. Theory and behavior of first price auctions. Am Econ Rev 79: 749762.

Harrison, G.W., 1994. Expected utility theory and the experimentalists. Empirical Econ 19: 223-253.

Hsu, M., Bhatt, M., Adolphs, A., Tranel, D., Camerer, C.F., 2005. Neural systems responding to degrees of uncertainty in human decision making. Science 5754:16801683.

Huettel, S.A., Stowe, C.J., Gordon, E.M., Warner, B.T., Platt, M.L., 2006. Neural signatures of economic preferences for risk and ambiguity. Neuron 49: 765-775.

Huettel, S.A., Song, A.W., McCarthy, G., 2005. Decisions under uncertainty: probabilistic context influences activation of prefrontal and parietal cortices. The Journal of Neuroscience 25: 3304-3311.

Johnson, E.J., Payne, J.W. 1985. Effort and accuracy in choice. Manage Sci 31: 395-414.

Kable, J.W., Glimcher, P.W., 2007. The neural correlates of subjective value during intertemporal choice. Nat Neurosci 10: 1625-1633.

Kahneman, D., Tversky, A., 1979. Econometrica 47:263-291.

Knutson, B., Fong, G.W., Adams, C.M., Varner, J.L., Hommer, D., 2001. Dissociation of reward anticipation and outcome with event-related fMRI. Neuroreport 12: 3683-3687.

Krajbich I, Armel C, Rangel A., 2010. Visual fixations and the computation and comparison of value in simple choice. Nat Neurosci 13: 1292-8

Krajbich I, Rangel A., 2011. Multialternative drift-diffusion model predicts the relationship between visual fixations and choice in value-based decisions. Proc Natl Acad Sci U S A 108: $13852-7$

Levy, I., Snell, J., Nelson, A.J., Rustichini, A., Glimcher, P.W., 2010. Neural representation of subjective value under risk and ambiguity. J Neurophysiol 103: 1036-1047.

Logan, G. D., Zbrodoff, N.J., 2003. Subitizing and similarity: Toward a pattern-matching theory of enumeration, Psychonomic Bulletin \& Review 10 : 676-682. 
Luce, R.D., 2000. Utility of Gains and Losses. Scientific Psychology Series. L. Erlbaum Associates, Mahwah, NJ, Vol 8.

MacCrimmon, K.R., Stanbury, W.T., Wehrung, D.A., 1980. Cognitive Processes in Choice and Decision Behavior. In: Wallsten T.S. (Eds.), L. Erlbaum Associates, Hillsdale, NJ.

Mosteller, F., Nogee, P., 1951. An experimental measurement of utility. J Polit Econ 59: 371404.

Moyer, R.S., Landauer, T.K., 1967. Nature 215: 1519:1520.

Nieder, A., Miller, E., 2003. Coding of cognitive magnitude: compressed scaling of numerical information in the primate prefrontal cortex. Neuron 37: 149-157.

Penny, W., Holmes, A., Friston, K., 2003. Random-effects analysis. In: Frackowiak R.S.J. et al. (eds), Human brain function. 2nd ed. London: Academic Press.

Padoa-Schioppa, C., Assad, J.A., 2006. Neurons in the orbitofrontal cortex encode economic value. Nature 441: 223-226.

Ratcliff, R., 1978. A theory of memory retrieval. Psychol Rev 85 :59-108.

Roland, P., Friberg, L., 1985. Localization of cortical areas activated by thinking. J. Neurophysiology 53: 1219-1243.

Rustichini, A., Dickhaut, J., Ghirardato, P., Smith, K., Pardo, J.V., 2005. A brain imaging study of the choice procedure. Games and Economic Behavior 52:257-282.

Sawamura, H., Shima, K., Tanji, J., 2002. Numerical representation for action in the parietal cortex of the monkey, Nature 415, 918-22.

Shadlen, M.N., Newsome, W.T., 1996. Motion perception: Seeing and deciding. Proc Natl Acad Sci USA 93: 628-633.

Siegel, S., 1964. Choice, Strategy and Utility. New York, McGraw-Hill.

Siegel, S., Messick, S., Brayfield, A.H., 1964. Decision and choice: Contributions of Sidney Siegal. New York: McGraw-Hill.

Smith, V.L., 1982. Microeconomic systems as an experimental science. Am Econ Rev 72: 923-955. 
Smith, V.L., Walker, J.M., 2007. Monetary rewards and decision cost in experimental economics. Econ Inquiry 31: 245-261.

Talairach, J., Tournoux, P., 1988. Co-planar stereotactic atlas of the human brain. Stuttgart: Georg Thieme.

Thurstone, L.L., 1931. The indifference function. J Soc Psychol 2: 139-167.

Tom, S.M., Fox, C.R., Trepel, C., Poldrack, R.A., 2007. The neural basis of loss aversion in decision-making under risk. Science 26(315): 515-518.

Tudusciuc, O., Nieder, A. 2007. Neuronal population coding of continuous and discrete quantity in the primate posterior parietal cortex. Proc Natl Acad Sci USA 104:1451314518.

Tudusciuc, O., Nieder, A., 2010. Comparison of length judgments and the Müller-Lyer illusion in monkeys and humans, Experimental Brain Research 207 : 221-31.

Yang, T., Shadlen, M.H., 2007. Probabilistic reasoning by neurons. Nature 447: 1075-1080. 


\section{Figure 1: Information processing and choice determination}

Figure 1.A is a description of an assumed theoretical distribution of the comparison metric, A, theorized to be internally generated by the subject (its exact form is not known). The metric when is converted to a signal, $y$, which either matches the statement that $\left(\theta_{1}-\theta_{2}\right)$ is greater than 0 , resulting in a signal 1 or the statement $\left(\theta_{1}-\theta_{2}\right)$ is not greater than 0 resulting in the signal -1. Figure 1.B indicates what happens to the distribution of the signal with increased effort. It shrinks the distribution leading to the conclusion that it is more likely a match will be detected when in fact there is a match. Figure 1.C the marginal costs of effort moving from southeast to northwest and the marginal benefits of effort moving from northeast to southwest. When a subject has greater experience the marginal benefit from effort shifts to the left because there are higher benefits to making efforts elsewhere. With lower quality information the cost of processing increases and reduces the efforts expended in the task. 
A.

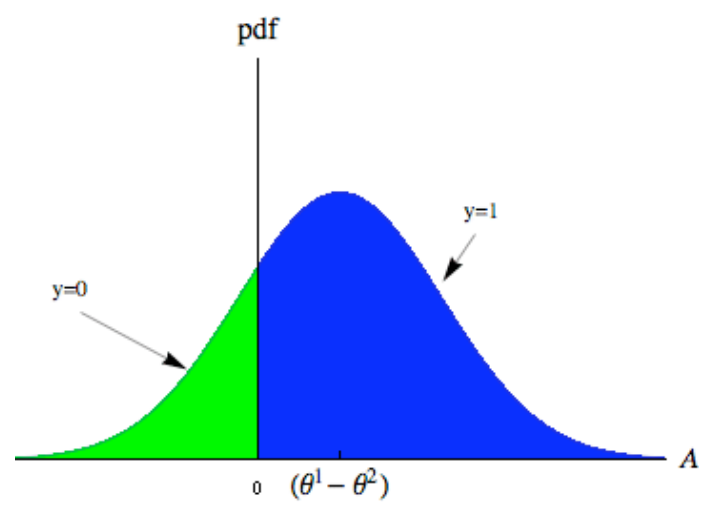

B.

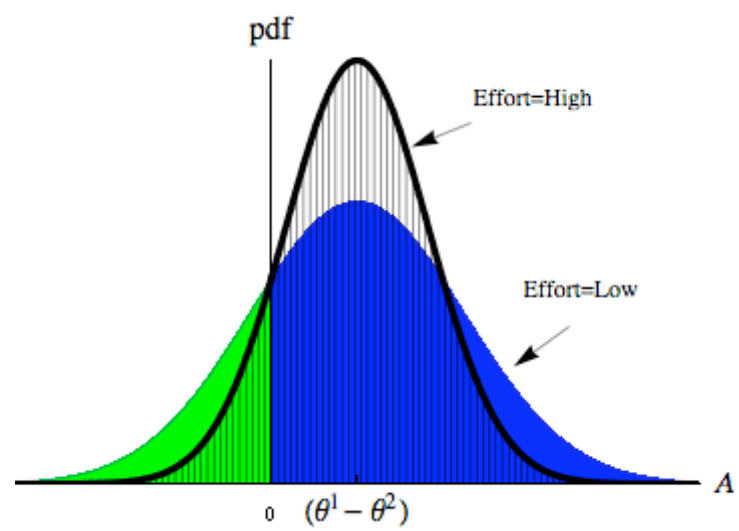

c.

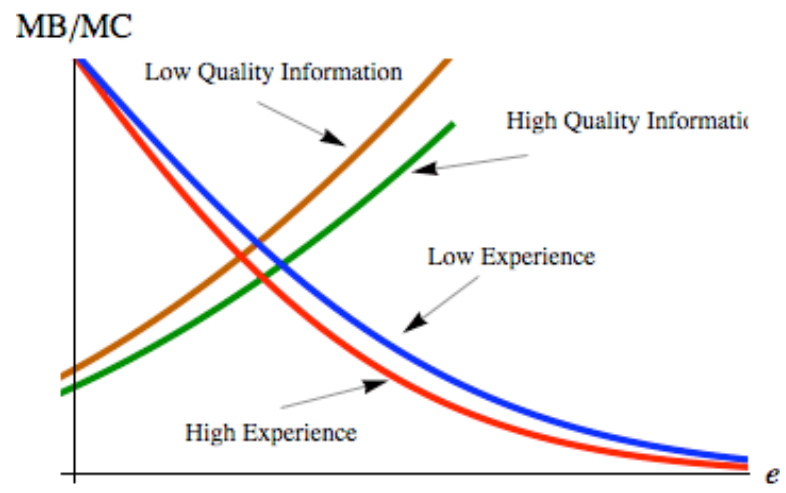




\section{Figure 2: Experimental design.}

The tasks in the behavioral and imaging experiment were identical. The experiment consisted of three blocks. Each block consisted in turn of fourteen periods. In each period, the subject had a choice between two options. One of the options was a lottery while the other was a certain amount. A lottery consists of two monetary prizes. Seven of the lotteries were risky, and seven ambiguous.

Panel A. The certain amount was one out of the set of seven elements 15, 17, 20, 23, 25, 27 and 30 dollars. The risky lottery consisted of a 50 per cent probability of 10 dollars (red area of the spinner) and a 50 percent probability of 50 dollars (green area of the spinner). The ambiguous lottery presented the payoffs for red and green outcomes, but the subject was not told the proportion of the two areas.

Panel B. A risky (ambiguous) choice is the choice between the risky (ambiguous) lottery and a certain amount. The risky lottery and the seven certain amounts lead to seven risky choices; similarly there are seven ambiguous choices. The right or left position of the lottery was randomly determined in each trial.

Panel C. The 14 stimuli were presented in three separate blocks.

Blocks 1 and 3 were identical. Each trial in these blocks had a display period that lasted 5 seconds, a choice period in which the subject could indicate a preferred option, with no time constraint, and an after-choice period, which lasted 13 seconds. In these blocks, the subject received no intermediate feedback.

In Block 2 the order of stimulus presentation was similar to the one in Blocks 1 and 3: in this block however the subject received information on the proportion of green and red in the ambiguous lotteries and then on the outcome of the uncertain lotteries. For ambiguous choices the proportions of green and red was revealed by the display of a pie chart identical to the one used to present risky lotteries. So in Block 2, after the subject had made the choice, the proportion of green and red areas was presented when the uncertain lottery was ambiguous, and then a spinner was spun superimposed on the pie chart representing the uncertain lottery: the rest position of the spinner indicated the outcome for that lottery. The overall spin time was 10 seconds. Following the spin there was a 13 second after-choice period as in Block 1 and 3. 


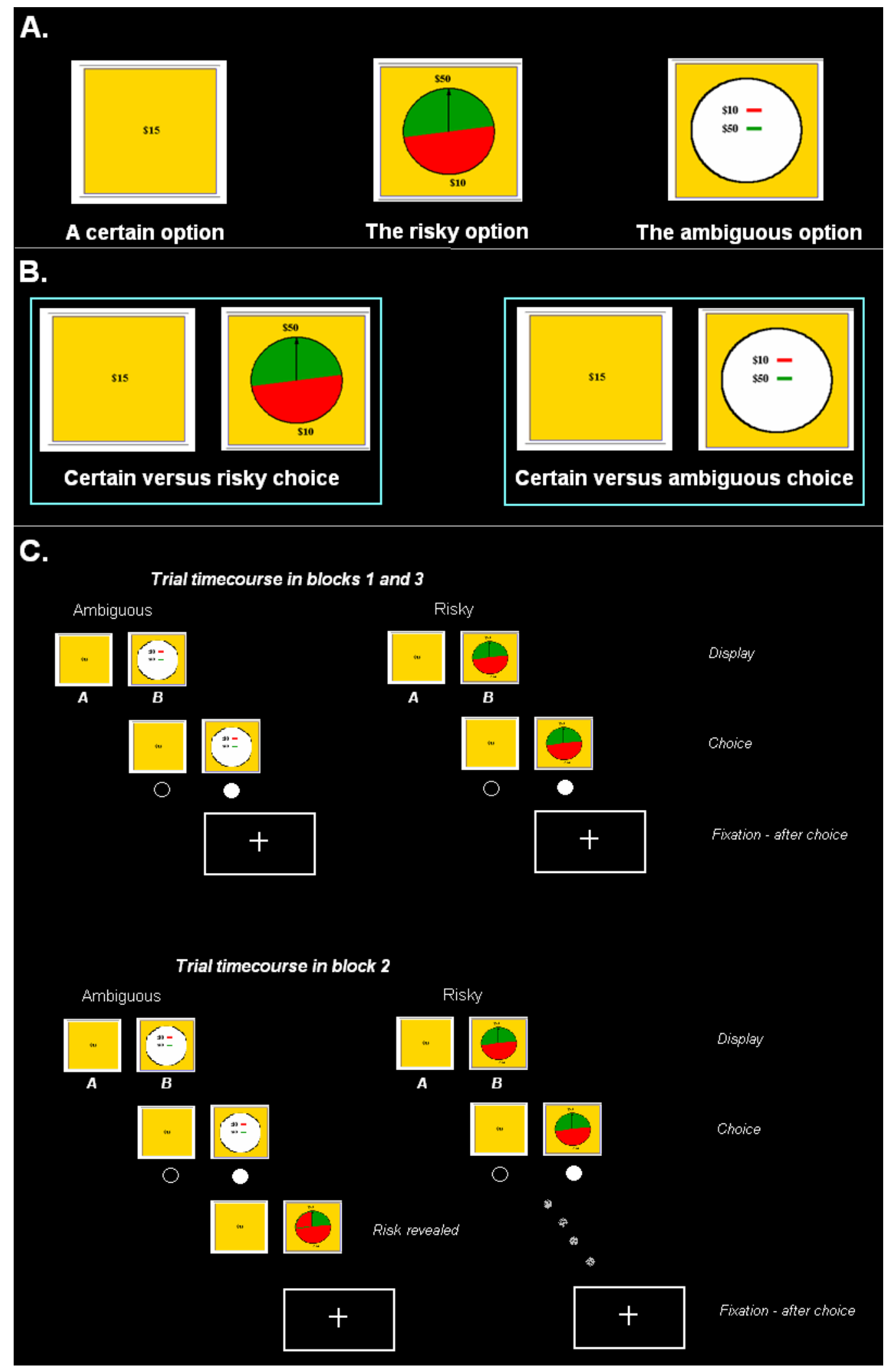




\section{Figure 3: Error rate, response time and distance from the certainty equivalent}

The left panel displays the lowess-smoothed error rate, bandwidth 0.8. In lowess, values are calculated by running a regression of the Error Rate on dummy variables indicating the value of the difference, values varying from 1 to 15 . The predicted values are calculated using a normally distributed density function $f$, and the corresponding solutions for error rate (the formula used to simulate the effort rate is:

$$
q_{z}=\frac{(t / p)^{2 z}-(t / p)^{z}}{(t / p)^{2 z}-1}
$$

where $p=P(1 \mid \theta, e), t=1-p$, and $z$ is the random walk parameter. See Dickhaut et al. 2009 for details). The right panel reports the scatterplot of the response time over all subjects.
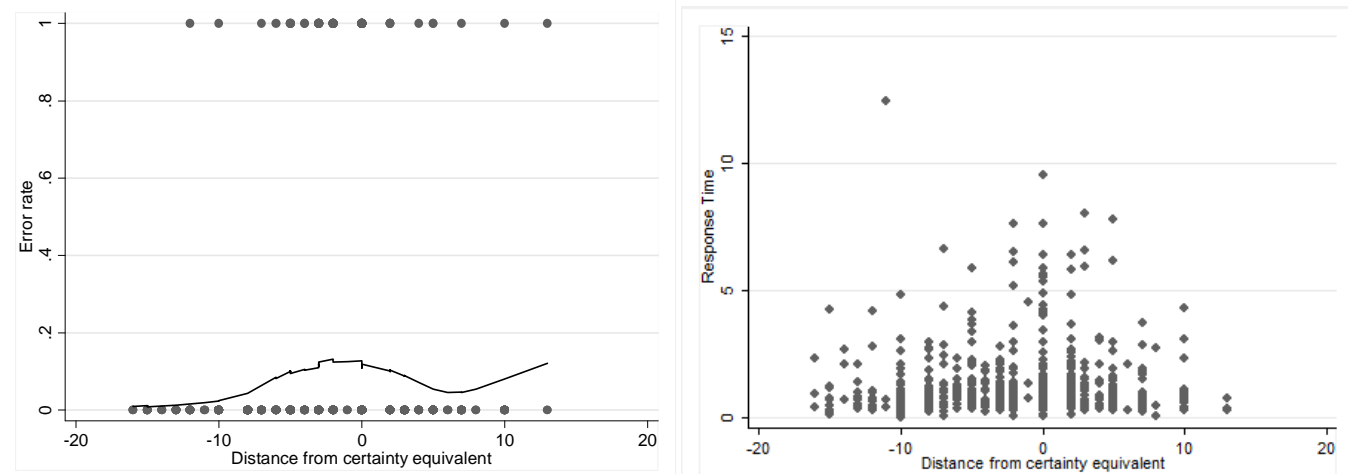
Figure 4: Kernel estimate of the response time in first and last block. The response time is considerably shorter in the last block (mean for block $1=1.48 \mathrm{~s}$, SE 0.11 ; mean for block $3=0.99$ s, SE 0.06.)

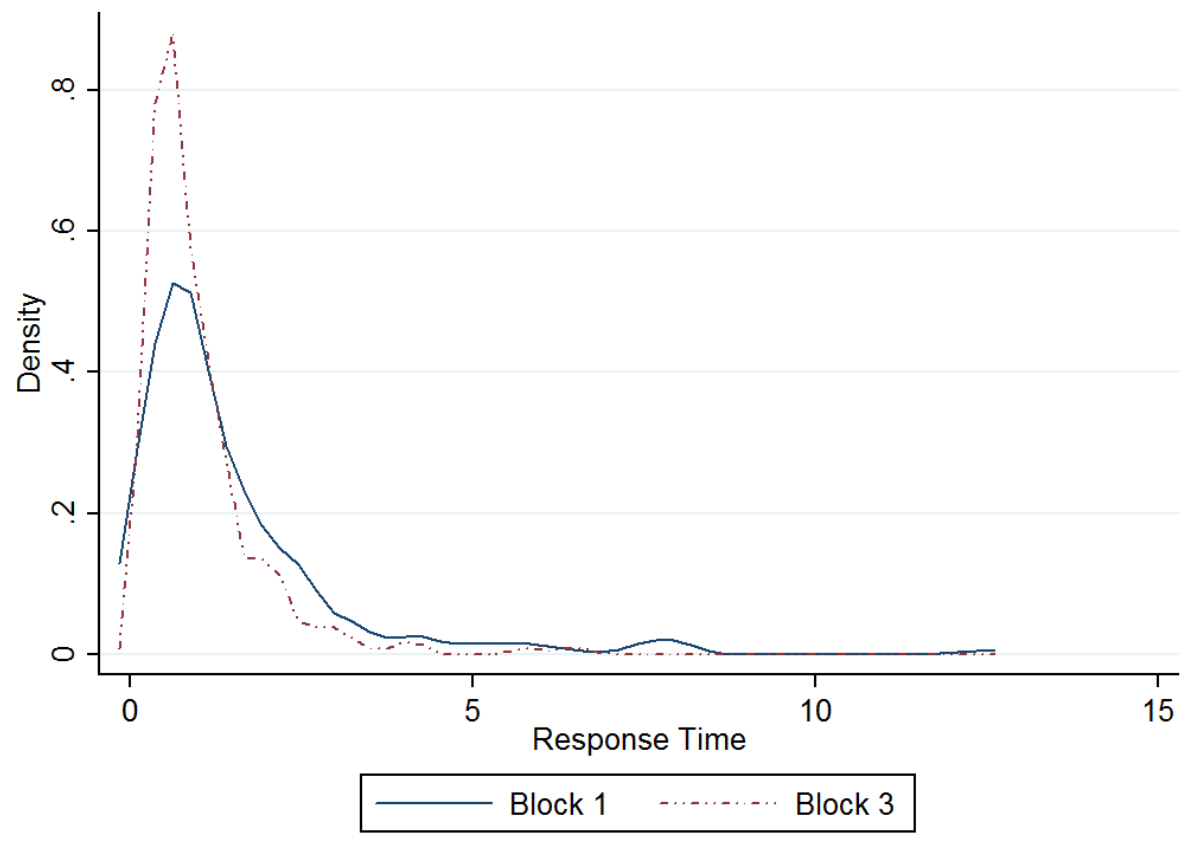




\section{Figure 5: Activation at Display and After Choice.}

Top panel. Areas that yielded a higher or lower activation during the display period than after the choice has been made. Data include all blocks. The color codes are reported on the right side of the figure. Higher activation was observed in two main areas of interest: bilaterally in the intraparietal sulcus, IPS, in the left hemisphere, $[-39,-38,+44]$, shown in the figure, and [+32, $-49,+40]$, not shown in the figure, and medial frontal gyrus, (MFG), encompassing supplementary motor area (SMA) and anterior cingulate cortex $[+8,0,+50]$. The IPS houses the neuronal representation of the ordered line segment. The cingulate and SMA are often found to be responsible for integrating various aspects of the choice process. Higher activation was observed in the orbito-frontal cortex (OFC), $[-3,+42,-8]$ in after choice than in display, which suggests a predominant evaluative role associated with the after-choice step.

Bottom panel. Event Related Averaging (ERA) of the time course of the BOLD signal timelocked to the onset of the stimulus onset, for the two stimuli, Display and After Choice. Note that the comparison is between events occurring at different times in the same trial. The bars report the 95\% confidence interval. The ERA's are displayed for the clusters in IPS, MFG and OFC described above.
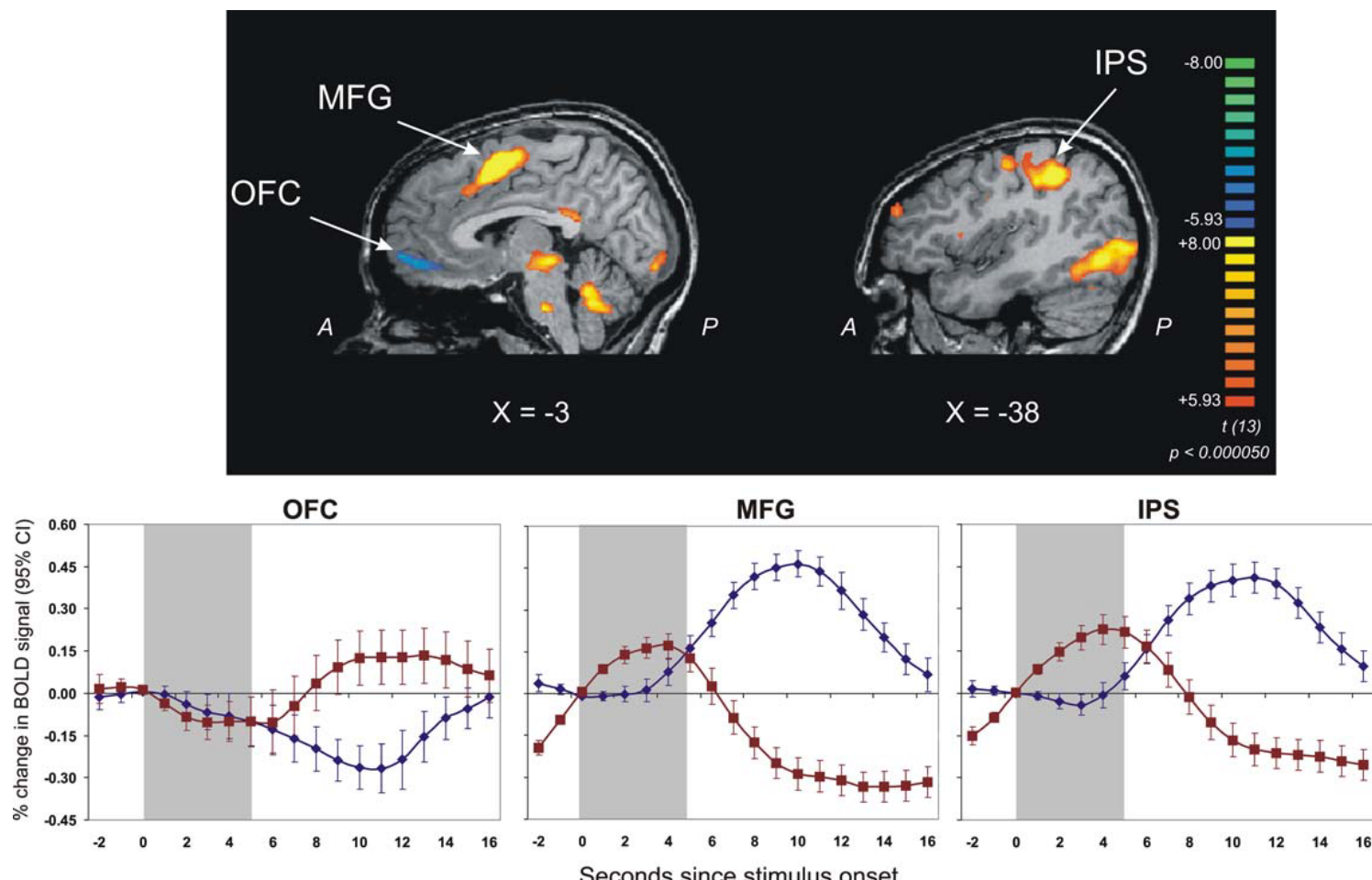

$\rightarrow$ Display $\rightarrow$-After choice 


\section{Figure 6: Risk, Ambiguity and distance from the cutoff.}

Top panel: Clusters of activation in the contrasts Display versus AfterChoice, in the two conditions of risky and ambiguous choices. The model is a parametric model with independent variables the distance of the certain amount from the certainty equivalent, the values of the certain amount, the number of choices made by the subject and the ambiguous choice. Data include all blocks. The color codes are reported on the right side of the figure. The pattern of activation is similar in the two conditions, with the size of the clusters and the maximum value of the $t$-statistic both larger in the condition of risky choice.

Bottom panel: The bar graphs display the average value of the coefficient of the four variables (distance of the certain amount from the cutoff, certain amount, number of choices made by the subject, and ambiguous choice), with one standard error super-imposed, for the four clusters identified in the Panel A: right and left IPS, Middle Frontal Gyrus (MFG) and Orbito-frontal (OFC). The coefficients were obtained by first estimating the percentage BOLD change (PBC) for the each of the 42 trials (the experiment had 3 blocks of 14 trials each), obtained by taking the event display in each trial as a distinct predictor. We then ran a regression on PBC with respect to the following variables: distance of the certain amount from the cutoff, certain amount, ambiguous and number of choices made by the subject. The four variables are normalized to have range between 0 and 1 , so the coefficients are directly comparable. The sign of the coefficients are as predicted by the model. Ambiguity has a negative effect. In addition, experience reduces the activation, as indicated by the negative value of the coefficient for the number of choices made.

The effect of the distance from the cutoff is negative: when the value is higher than the cutoff, the activation is reduced. This occurs in all four regions, including the OFC, in a significant way. The effect of the certain amount is positive. Both coefficients are strongly significant for the two IPS regions ( $p=0.0001$ for the Left IPS). 

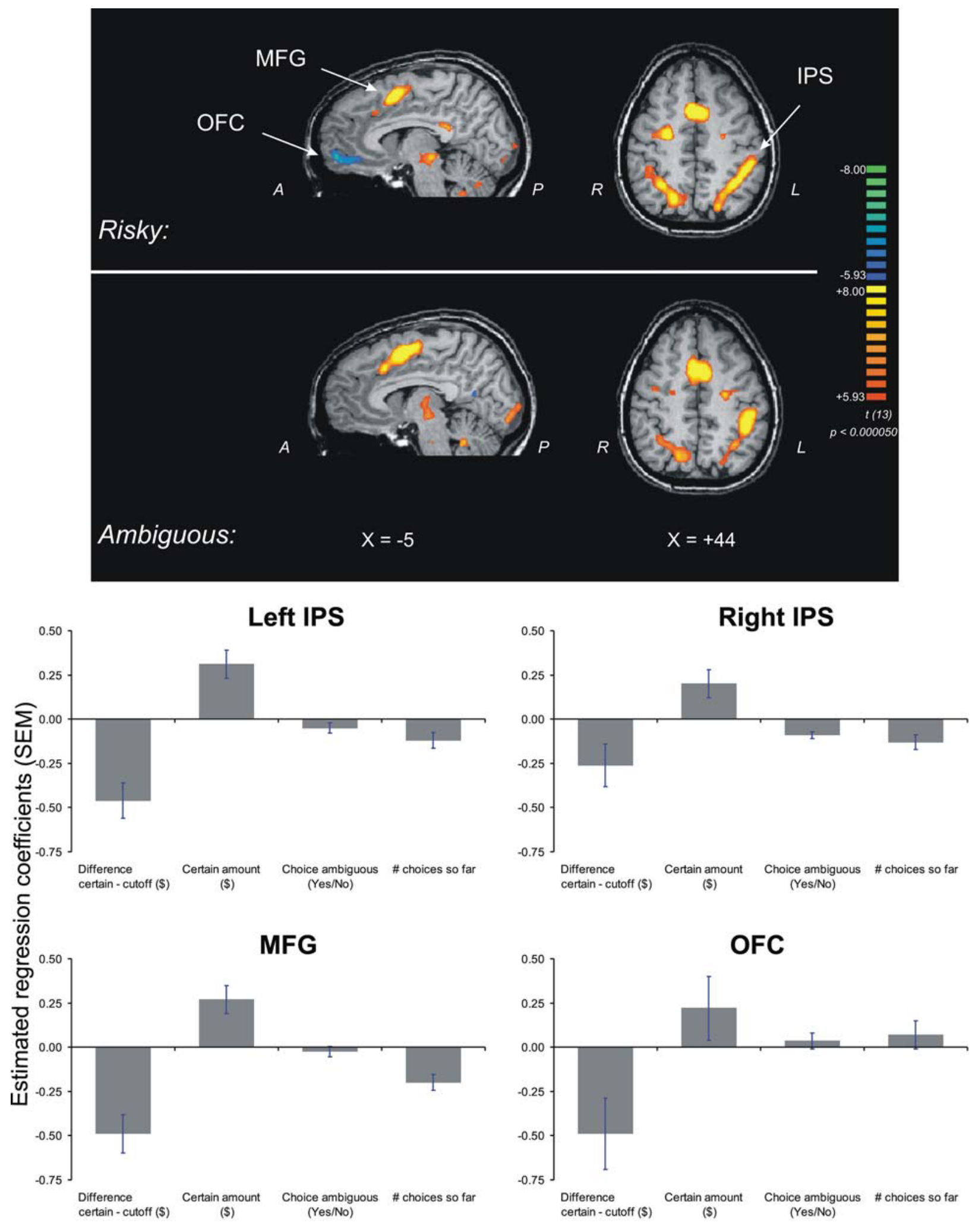


\section{Regressions}

\section{Table 1: Panel data logit regression of the choice of the certain amount}

The regressions are Fixed effects. The table reports estimated coefficients; odd ratios are reported in the text. The $p$-value is reported in parentheses. Here and below: *** denotes $p<0.01, * * p<0.05, * p<0.1$. The variable ambiguous is equal to 1 if the choice was ambiguous; Certain: value of the certain amount in that trial, in dollars; Cumulative choices: the total choices made at the moment of the trial by the subject.

\begin{tabular}{lccc} 
VARIABLES & Ch. certain & Ch. certain & Ch. certain \\
\hline Ambiguous & 0.270 & $0.465^{* *}$ & $0.447^{*}$ \\
Certain & $(0.120)$ & $(0.0425)$ & $(0.0520)$ \\
& & $0.388^{* * *}$ & $0.391^{* * *}$ \\
Cumulative choices & & $(0.000)$ & $(0.000)$ \\
& & & $-0.0173^{*}$ \\
Observations & & & $(0.0672)$ \\
Number of subjects & 588 & 588 & 588 \\
\hline
\end{tabular}


Table 2: Panel data logit regression of the Error rate Fixed effects. Variables are as described in Table 1. Two of the subjects had no error.

\begin{tabular}{lccc}
\multicolumn{1}{c}{ VARIABLES } & Error & Error & Error \\
\hline Ambiguous & -0.383 & -0.390 & -0.429 \\
& $(0.220)$ & $(0.215)$ & $(0.182)$ \\
Certain & & $0.0822^{* *}$ & $0.0836^{* *}$ \\
& & $(0.0112)$ & $(0.0114)$ \\
Cumulative choices & & $-0.0460^{* * *}$ \\
& & & $(0.000816)$ \\
Observations & 504 & 504 & 504 \\
Number of subjects & 12 & 12 & 12 \\
\hline
\end{tabular}


Table 3: Panel data regression of the Response Time Fixed effects Variables are as described in Table 1.

\begin{tabular}{lccc} 
VARIABLES & Response Time & Response Time & Response Time \\
\hline Ambiguous & -0.172 & -0.172 & $-0.201^{*}$ \\
Certain & $(0.104)$ & $(0.101)$ & $(0.0512)$ \\
& & $0.0359^{* * *}$ & $0.0360^{* * *}$ \\
Cum. choices & & $(0.000641)$ & $(0.000489)$ \\
& & & $-0.0216^{* * *}$ \\
Constant & $1.400^{* * *}$ & $0.594^{* *}$ & $(5.00 \mathrm{e}-07)$ \\
& $(0.000)$ & $(0.0162)$ & $1.051^{* * *}$ \\
Observations & & & $(5.08 \mathrm{e}-05)$ \\
Number of subjects & 588 & 588 & 588 \\
\hline
\end{tabular}

